Review

\title{
Clostridium perfringens Iota-Toxin: Structure and Function
}

\author{
Jun Sakurai $^{1, *}$, Masahiro Nagahama ${ }^{1}$, Masataka Oda ${ }^{1}$, Hideaki Tsuge ${ }^{2}$ and Keiko Kobayashi ${ }^{1}$ \\ 1 Department of Microbiology, Faculty of Pharmaceutical Sciences, Tokushima Bunri University, \\ Yamashiro-cho, Tokushima 770-8514, Japan \\ 2 Institute for Health Sciences, Tokushima Bunri University, Yamashiro-cho, Tokushima 770-8514, \\ Japan \\ * Author to whom correspondence should be addressed; E-Mail: sakurai@ph.bunri-u.ac.jp; \\ Tel.: +8-188-602-8483; Fax: +8-188-655-3051.
}

Received: 27 November 2009; in revised form: 16 December 2009 / Accepted: 21 December 2009 / Published: 23 December 2009

\begin{abstract}
Clostridium perfringens iota-toxin is composed of the enzyme component (Ia) and the binding component (Ib). Ib binds to receptor on targeted cells and translocates Ia into the cytosol of the cells. Ia ADP-ribosylates actin, resulting in cell rounding and death. Comparisons of the deduced amino acid sequence from the gene and three-dimensional structure of Ia with those of ADP-ribosylating toxins (ARTs) suggests that there is striking structural similarity among these toxins. Our objectives are to review the recent advances in the character, structure-function, and the mode of action of iota-toxin by consideration of the findings about ARTs.
\end{abstract}

Keywords: Clostridium perfringens; iota-toxin; ADP-ribosylating toxin; $\mathrm{NAD}^{+}$-glycohydrolase; ADP-ribosyltransferase; crystal structure; SN1 mechanism; internalization; endocytosis

\section{Introduction}

Clostridium perfringens is rod-shaped and gram positive. The microorganism is an anaerobic bacterium, but not a strict anaerobe. Strains of $C$. perfringens produce four major protein toxins, called alpha-, beta-, epsilon- and iota-toxins, which possess lethal and dermonecrotic activities at least, and 
are classified into five groups (type A to E) [1-3]. C. perfringens type E, which produces alpha- and iota-toxins, causes antibiotic-associated enterotoxemia in rabbits, and is also implicated in sporadic outbreaks among calves as well as lambs [4]. C. spiroforme iota-like toxin is reported to be closely related to enteritis in rabbits [4]. Therefore, iota-toxin is highly likely to an important agent of enterotoxemia caused by $C$. perfringens type $\mathrm{E}$.

Iota-toxin composed of an enzyme component (Ia) and a binding component (Ib) is a member of the binary toxin group $[2,3,5,6]$ (Tables 1 and 2). Significant progress has been made in the characterization of iota-toxin [5]. In addition, information on the biological properties, structure-function and mode of action of iota-toxin also has been accumulated [5]. This article summarizes current findings and deals with the mechanism of iota-toxin.

Table 1. Characterization of Clostridium perfringens iota-toxin.

\begin{tabular}{ll}
\hline Molecular weight & Iota a (Ia) 47,605 Da (413 residues) \\
& Iota b (Ib) 74,147 Da (664 residues) \\
Biological activity & Lethality, Dermonecrosis, Cytotoxicity \\
Enzymatic activity of Ia & NAD $^{+}$-glycohydrolase (NADase) \\
& ADP-ribosyltransferase (ARTase) \\
\hline
\end{tabular}

Table 2. Structural organization of the binary toxin family.

\begin{tabular}{lcc}
\hline Toxin & Enzymatic component & Binding component \\
\hline C. perfringens Iota-toxin & $\mathrm{Ia}$ & $\mathrm{Ib}$ \\
B. anthracis Toxin & Lethal factor & Protective antigen \\
& Edema factor & \\
C. botulinum C2toxin & $\mathrm{C} 2 \mathrm{I}$ & $\mathrm{C} 2 \mathrm{II}$ \\
C. spiroforme Iota-like toxin & $\mathrm{Sa}$ & $\mathrm{Sb}$ \\
\hline
\end{tabular}

\section{Characteristics of Iota-Toxin}

C. perfringens iota-toxin was initially described by Bosworth in 1943 [7]. Later, the iota toxin was found to consist of two components [8], encoded by two genes in a plasmid, organized in an operon [9]. Two genes, with the same orientation, coding for Ia (454 amino acids) and Ib (875 amino acids) and separated by 243 noncoding nucleotides, were identified. It was reported that a predicted signal peptide (41 amino acids) and propeptide (13 amino acids) are missing from Ia and a predicted signal peptide (39 amino acids) and propeptide (211 amino acids) are missing from Ib [10], suggesting that the active Ia and $\mathrm{Ib}$ are composed of 400 and 664 amino acid residues, respectively. Alone, each component is nontoxic, but together, Ia and $\mathrm{Ib}$ are cytotoxic to various cultured cells, lethal to mice and dermonecrotic in guinea pigs [1,2,11] (Table 1).

Ia ADP-ribosylates skeletal muscle $\alpha$-actin and nonmuscle $\beta / \gamma$-actin [2,3] (Figure 1). ADP-ribosylating toxins facilitate scission of the $N$-glycosyl bond between nicotinamide and the $N$-ribose of NAD (NAD ${ }^{+}$-glycohydrolase, NADase) and transfer the ADP-ribose moiety to target 
proteins (ADP-ribosyltransferase, ARTase) [5]. ADP-ribosylating toxins are classified into four families (Types I to IV) based on their respective targets [12,13] (Figure 2). Type I targets heteromeric GTP-binding proteins, and includes cholera toxin (CT) [14], pertussis toxin (PT) [15], and Escherichia coli heat-labile enterotoxin (LT) [16]. Type II [diphtheria toxin (DT) [17] and Pseudomonas exotoxin A (ETA) [18]] modifies elongation factor 2 (EF-2). Type III [C. botulinum C3 exoenzyme] ADP-ribosylates small GTP-binding proteins [19]. Type IV ADP-ribosylates actin [5]. Ia belongs to Type IV. Iota-toxin is a member of the binary toxin group, which includes $C$. botulinum $\mathrm{C} 2$ toxin (C2I and C2II) [20], C. difficile ADP-ribosyltransferase (CDTa and CDTb) [21], C. spiroforme toxin (Sa and $\mathrm{Sb}$ ) [22] and Bacillus cereus vegetative insecticidal protein (VIP) [5]. The enzyme components of these toxins, which are structurally similar to Ia, are organized according to the classic A-B model.

Figure 1. ADP-ribosylating activity of Ia.

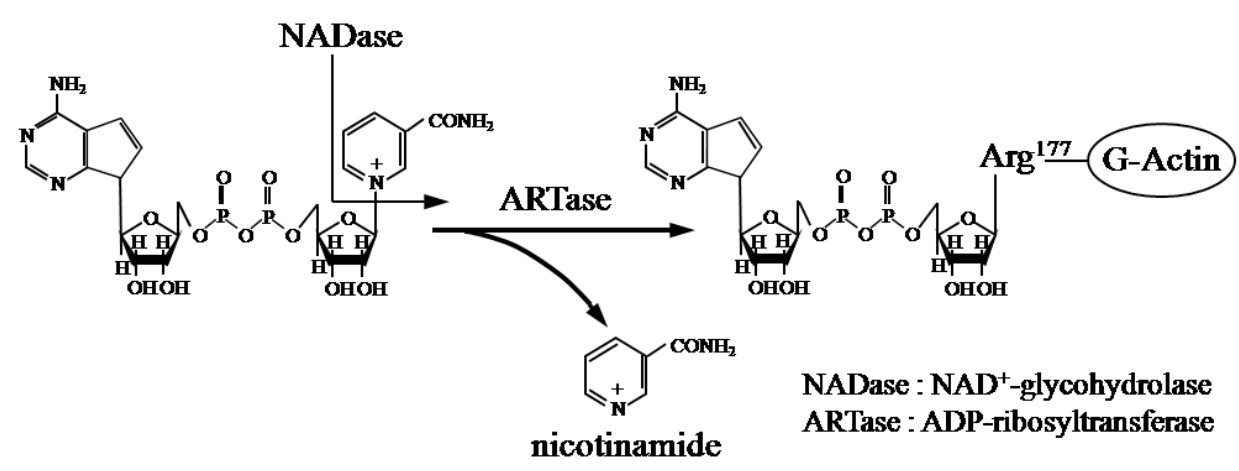

Figure 2. A family of bacterial ADP-ribosylating toxins.

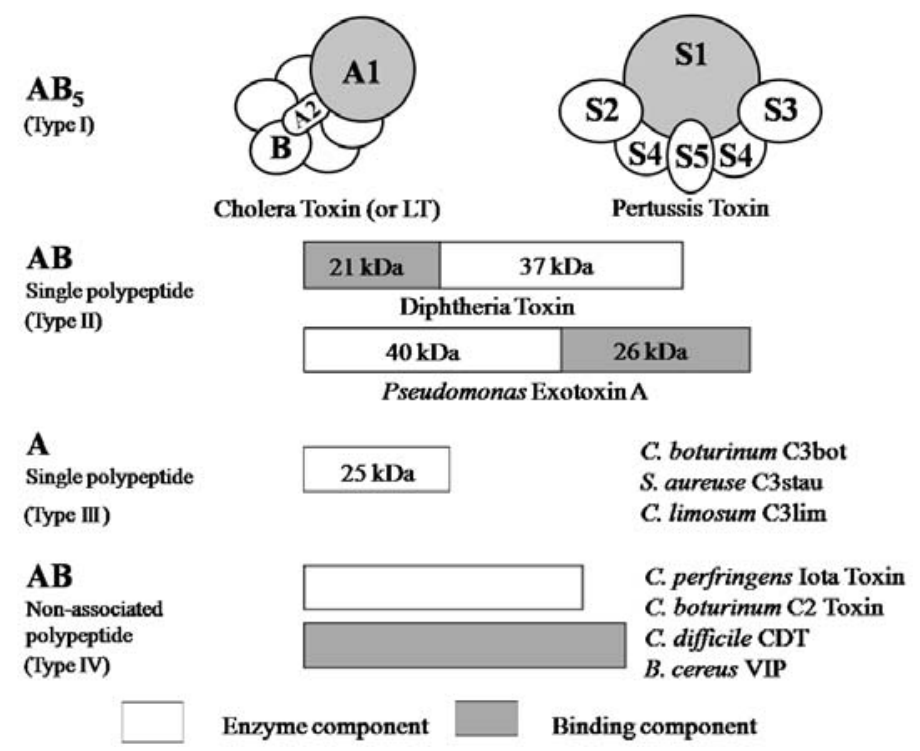

Ib displays significant homology with the protective antigen (PA) of anthrax toxins (54.4\% similarity overall) and C2II (39.0\% similarity overall) [5] (Figure 3). The binding components of iota-toxin, anthrax toxin and $\mathrm{C} 2$ toxin similarly bind to receptors on membranes and interact with the enzyme components, mediating their entry into target cells, suggesting that they have a similar mode 
of action. As shown in Figure 3, the numbers reveal good homology in domains I, II and III forming the pore. In contrast, the homology in the receptor-binding domain IV is negligible among members of this group, which can be explained by the receptor-specificity of domain IV [5]. Therefore, the binding components of these binary toxins structurally seem to be conserved.

Furthermore, Richard et al. reported that iota-toxin applied apically or basolaterally induces a rapid decrease in the transepithelial resistance (TER) of monolayers of CaCo-2 cells and disorganization of actin filaments as well as the tight and adherens junctions [23]. Treatment of Vero cells with iota toxin resulted in delayed caspase-dependent death. Unmodified actin did not reappear in toxin-treated cells, and Ia was detectable in the cytosol [24]. PA and C2II bind to receptors on cells and interact with edema factor or lethal factor and C2I, respectively, mediating entry into target cells [5]. Ib also binds to a receptor, interacts with Ia and transfers Ia into the cytosol. The activity of iota-toxin severely reduces the ability of actin to undergo polymerization, leading to disruption of the cytoskeletal architecture and cell death [5].

Figure 3. Comparison of amino acid sequence homology between Ib and PA or C2II.

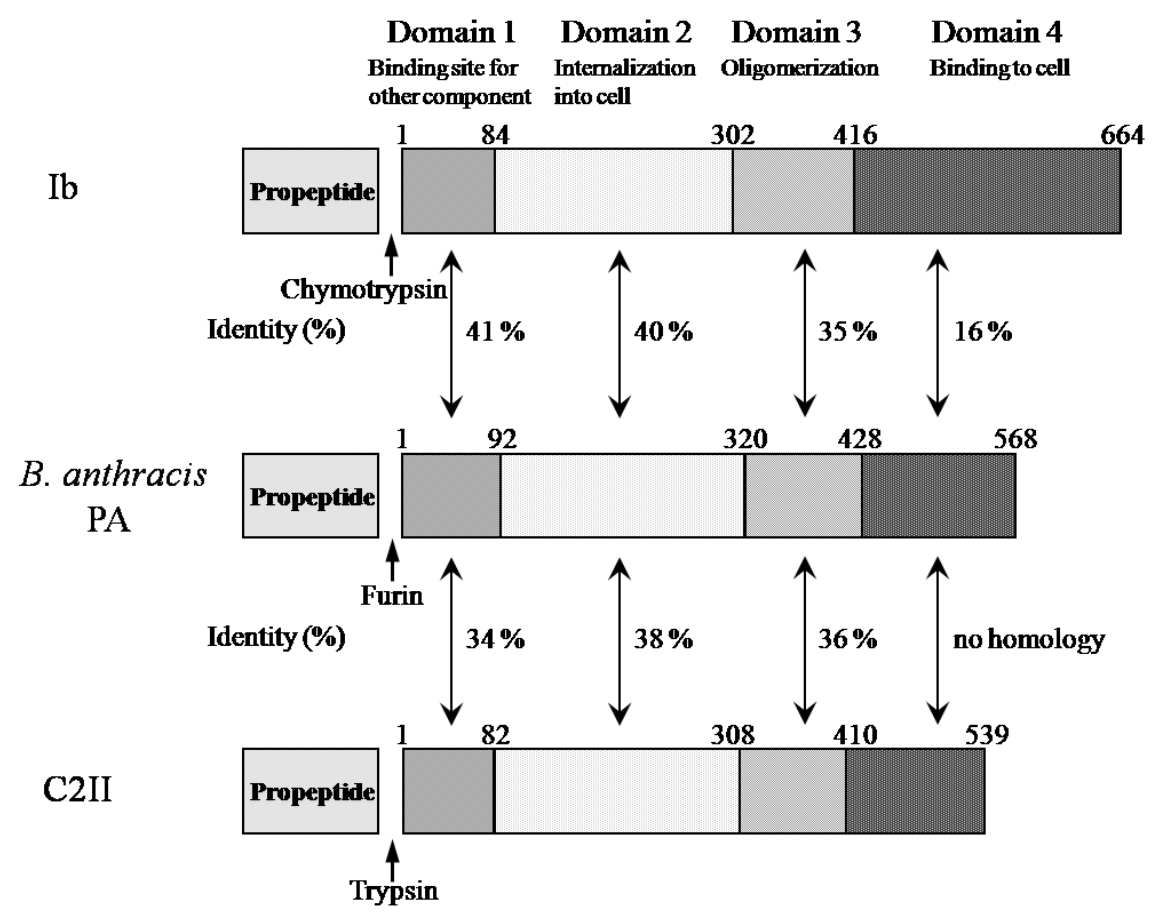

\section{Structure and Function}

Bacterial ADP-ribosylating toxins have been studied as agents that contribute to the pathogenesis of bacteria. Domenighini and Rappouli [25] classified ADP-ribosylating toxins into a CT group and a DT group, on the basis of amino acid sequences involved in the formation of the NAD-binding site and expected to play a role in the activity or in the folding of the proteins. In addition, the two groups do not share any detectable sequence homology.

Analysis of the LT crystal suggested that the nicotinamide ring of NAD ${ }^{+}$docks into the cavity [25]. ADP-ribosylating toxins of the CT group including LT, PT, B. sphaericus mosquitocidal toxin (MTX) 
[26], ExoS and C3 are known to contain three conserved regions, aromatic residue-R/H, E-X-E, and hydrophobic residue-S-T-S-hydrophobic residue, in the cavity formed by the $\beta / \alpha$ motif [25] (Figure 4). The role of these regions had been thought to be as follows. The polar side chains of E-X-E extend toward the catalytic cavity and are the common sequence involved in forming the $\mathrm{NAD}^{+}$cleft, an aromatic residue- $\mathrm{R} / \mathrm{H}$ located deep in the cavity binds $\mathrm{NAD}^{+}$, and the $\mathrm{S}-\mathrm{T}-\mathrm{S}$ consensus sequence is folded in a $\beta$ strand representing the floor of the cavity [25]. Glu-378 and -380 in Ia are included within the E-X-E sequence essential for the enzymatic activity of CT, LT, and ExoS. Arg-295 is present in the aromatic residue-R/H sequence. Ser-338, Ser-340, and Thr-339 are present in the S-T-S consensus sequence [25]. A comparison of the amino acid sequence of Ia with sequences of these ADP-ribosylating toxins confirmed the existence of these motifs in Ia, showing that Ia belongs to the CT group.

Figure 4. Conserved regions in ADP-ribosylating toxins.

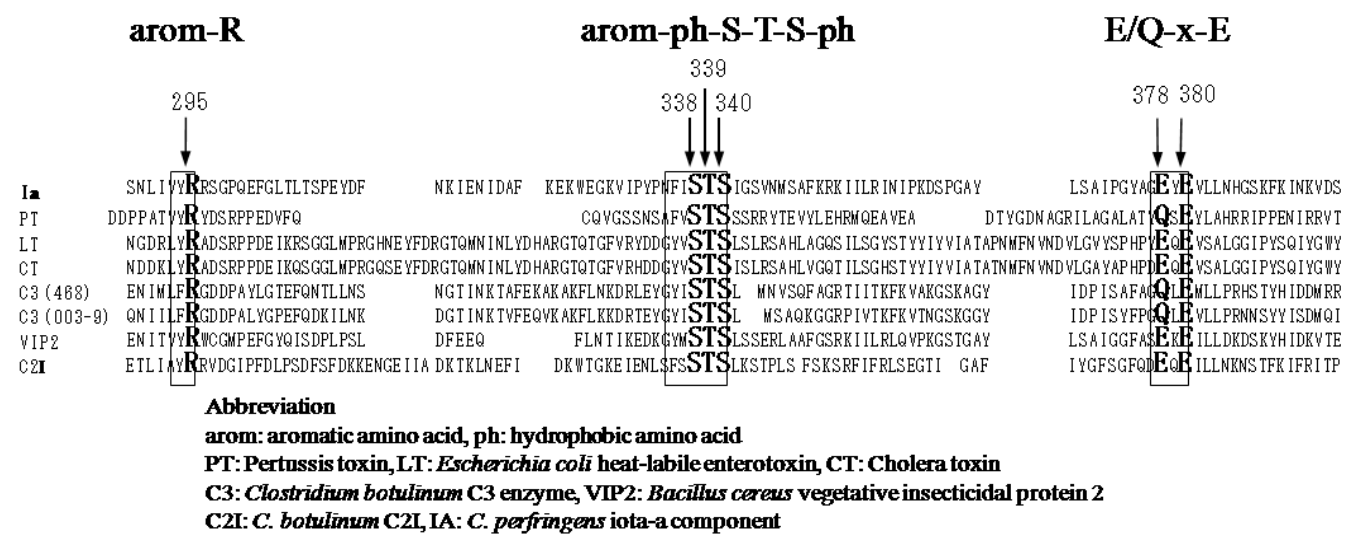

Replacement of Arg-295 with alanine in Ia led to a complete loss of NADase, ARTase, lethal, and cytotoxic activities [27]. Perelle et al. [28] also reported that this residue is essential for ADP-ribosylating activity. Thus, it appears that the Arg-295 of Ia is equivalent to the Arg-7 of LT and Arg-299 of C2 toxin, which are required for ADP-ribosylating activity [29,30]. Replacement of Arg-295 with a basic amino acid such as histidine or lysine also caused a drastic reduction in NADase and/or ARTase activities, showing that Arg-295 can not be substituted with other basic residues. Therefore, it is apparent that the side chain of Arg-295 is essential for the NADase and ARTase activities of Ia [27]. Substitution of Arg-295 with lysine in Ia resulted in a significant reduction in the $K m$ for $\mathrm{NAD}^{+}$, markedly reduced the $k c a t$ values for NADase and ARTase, but had little effect on the $K m$ for actin [27]. Accordingly, these observations suggest that Arg-295 plays an essential role in the binding of Ia to NAD ${ }^{+}$.

Substitution of Glu-378 within the E-X-E sequence with alanine in Ia resulted in a complete loss of NADase and ARTase activities [27], as reported by Perelle et al. [28]; however, conservative substitution of Glu-378 with aspartic acid resulted in little effect on NADase activity, and a drastic reduction in ARTase activity. These results indicate that Glu-378 plays an important role in ARTase activity, but not in NADase activity. It therefore is likely that the carboxyl group of the side chain in 
the amino acid at position 378 is not required for NADase activity, but is essential for ARTase activity. Barth et al. [29] and Radke et al. [31] found that Glu-378 within the E-X-E motif (Glu378-X-Glu379) of C2 toxin and Glu-379 within the motif Glu379-X-Glu381 of ExoS were essential for ARTase activity, but not required for NADase activity. Our results support these findings. A kinetic analysis showed that replacement of Glu-378 with aspartic acid resulted in a severe reduction in the kcat values for ARTase activity, but had little effect on the $\mathrm{Km}$ values for $\mathrm{NAD}^{+}$and actin, suggesting that the residue plays an important role in the catalytic mechanism. The glutamic acid at position 380 within the E-X-E sequence was replaced with aspartic acid and glutamine. The result shows that a conservative substitution, such as reduction of the carboxyl group at position 380 by one methylene unit or replacement of the carboxyl group by an uncharged amide, simultaneously resulted in a drastic reduction in NADase and ARTase activities. Furthermore, replacement of Glu-378 and Glu-380 with aspartic acid resulted in little effect on NADase activity and a drastic reduction in ARTase activity, respectively suggesting that Glu-380 is essential for the catalytic mechanism of ARTase. Thus, the role of Glu-380 in Ia appears to be equivalent to that of the corresponding residues in C2 toxin and LT [27]. Damme et al. [32] reported the Glu-378 in Ia was photolabeled by NAD ${ }^{+}$, but that Glu-380 was not. Therefore, the Glu-378 and -380 residues seem to play different roles in the ADP-ribosylating activity of Ia.

Cieplak et al. [33] reported that substitution of Glu-112 (E110-X-E112) in LT resulted in a marked reduction in ARTase activity, suggesting that the residue plays a specific role in the mechanism of ADP-ribosylation and represents an essential catalytic residue. In addition, they suggested that Glu-110 is unlikely to play a specific role in the reaction mechanism. Hara et al. [34] reported that rat T-cell antigen RT 6.1 (Q207-X-E209) catalyzes NADase, but not transfer of the ADP-ribosyl moiety and that a mutant RT 6.1 in which Gln-207 was replaced with glutamic acid exhibited ARTase activity. Our result is consistent with their findings in that the first glutamic acid residue in the E-X-E motif is essential for ARTase activity, but not for NADase activity [27]. However, C3 and EDIN in the ADP ribosyltransferase family, which ADP-ribosylates small GTP binding proteins of the rho family, have a glutamine residue in the motif, suggesting that the residue in C3 and EDIN which correspond to the residue at position 380 in Ia is not required for ARTase activity [35]. Thus, the residue in the motif may depend on the substrate.

The replacement of Ser-338 with alanine or cysteine was not entirely effective, suggesting that the hydroxyl group of Ser-338 is not essential for these activities [27]. Furthermore, the substitution of Ser-338 with amino acids having a large side chain completely disturbed the ARTase activity. Thus, Ser-338 may be extremely close to the catalytic site. Barth et al. [29] reported that Ser-348 in the motif $\mathrm{S} 348-\mathrm{T}-\mathrm{S} 350$ in $\mathrm{C} 2$ toxin plays an essential role in $\mathrm{NAD}^{+}-$binding or catalysis. There is no discrepancy between our results and their model. Replacement of Thr-339 or Ser-340 with alanine resulted in a significant but not severe reduction in NADase and ARTase activities. Replacement of Thr-339 and Ser-340 did not result in a marked reduction in the Km and kcat values for NADase or ARTase, compared with that of Ser-338. Therefore, it is possible that these residues do not play an important role in binding and catalytic reactions [27]. 
These observations indicate that Ia has both NADase and ARTase activities; the former transfers the ADP ribose moiety to a water molecule, and the latter transfers the moiety to actin [5]. Thus, it seems that Arg-298 and Glu-380, required for these activities in Ia, play an important role in the cleavage of the $N$-glycosidic bond of $\mathrm{NAD}^{+}$and that Glu-378, required for ARTase activity, but not for NADase activity, is essential for the transfer of the ADP-ribose moiety to actin, confirming that Ia belongs to the CT group in the ADP-ribosylating enzyme family, not the DT group [27] (Figure 5).

Figure 5. Amino acid residues at the active site of Ia.
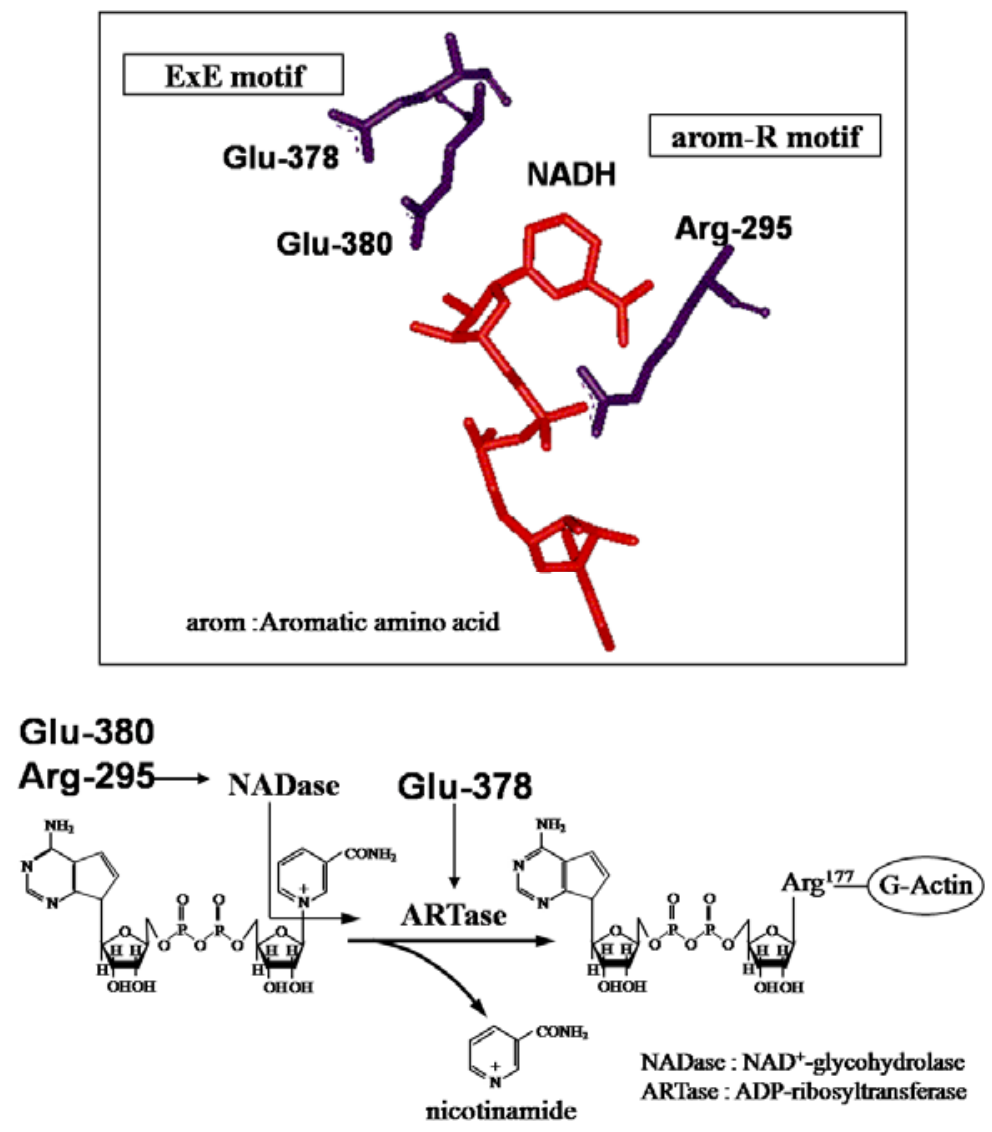

The crystal structure of PA [36] and C2II [37] similarly reveals four domains in these components. Gupta et al. [38] suggested that $\mathrm{Ca}^{2+}$ plays a role in maintaining the conformation of PA, based on an analysis of the relationship between $\mathrm{Ca}^{2+}$ and PA. Gao-Sheridan et al. [39] reported that the bound $\mathrm{Ca}^{2+}$ in PA plays a role in maintaining the conformation of an $N$-terminal region designated domain I which allows PA63 to oligomerize and bind to the enzyme components. It also is speculated that the $N$-terminal region of the binding component has five conserved Asp residues and one conserved Glu residue whose side-chain carboxyl groups chelate two calcium ions ( $\mathrm{Ca}^{2+}$-binding motif) [40] (Figure 6). It was reported that the $\mathrm{N}$ and $\mathrm{C}$ termini in the binding components represent the docking site for the enzyme component and the binding site for the cell, respectively [5]. We reported that $\mathrm{Ib}$ binds to a receptor on MDCK cells, forms a heptamer, and then the oligomer is internalized via endocytosis [41]. The replacement with alanine of Asp-8, -10 and -12, which are included within the conserved motif in $\mathrm{Ib}$, led to a severe reduction in cytotoxic activity [42]. The internalization of these variants in cells without Ia resembled that of wild-type Ib alone [42]. We also reported that Ia bound to 
$\mathrm{Ib}$ in lipid rafts is internalized by endocytosis [43]. However, Ia is not internalized in the cells in the presence of D8A, D10A and D12A, suggesting that the $\mathrm{Ca}^{2+}$-binding motif of $\mathrm{Ib}$ has an important role in the interaction of Ia in the presence of $\mathrm{Ca}^{2+}$ [42]. Gao-Sheridin et al. [39] reported that $\mathrm{Ca}^{2+}$ within domain I of PA is required for PA63 subunit-subunit interactions. Gupta et al. [38] speculated on the basis of the replacement of amino acid residues in the $\mathrm{Ca}^{2+}$-binding motif of domain I of PA, that the motif is critical for initial protein assembly. However, substitutions at Asp-8, -10 and -12 in Ib resulted in about half as much oligomer in cell membranes, indicating that the residues in the $\mathrm{Ca}^{2+}$ - binding motif of Ib did not greatly affect the oligomerization of $\mathrm{Ib}[42]$.

Figure 6. Amino acid sequence in the region containing the calcium-binding motif in members of the binary toxin family.

\section{Bacillus anthracis protective antigen (PA)

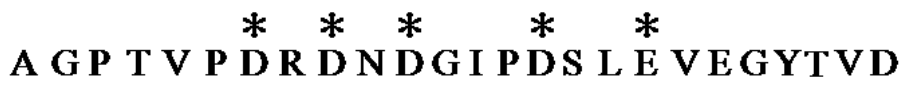 Clostridium perfringens Ib W E D E D L D T D N D N I P D A Y E K NGYT I K Clostridium botulinum C2 II K A N A N R D T D R D G I P DE WE I NGYTVM Clostridium difficile CDTb W E D E D L D T D N D N I P DS Y E R NGYT I K * : Residue involving $\mathrm{Ca}^{2+}$ chelator}

We solved the crystal structure of Ia with $\mathrm{NADH}$, which acts as a inhibitor of the enzymatic activity of Ia, at a resolution of $1.8 \AA[44,45]$. The 411 amino acid residues of Ia were folded into a mixed $\alpha / \beta$ structure of dimensions $68 \AA \times 50 \AA \times 47 \AA$. The enzyme is divided into two domains, the $N$-domain (residues 3-210) and the $C$-domain (residues 211-413) (Figure 7A). These domains are structurally similar despite limited sequence identity. Each domain consists of a $\beta$-sandwich core formed by a five-stranded mixed $\beta$-sheet $(\beta 1, \beta 4, \beta 8, \beta 7$, and $\beta 2$ in the $N$-domain; $\beta 9, \beta 12, \beta 16, \beta 15$, and $\beta 10$ in the $C$-domain) and a three stranded anti-parallel $\beta$-sheet $(\beta 3, \beta 6$, and $\beta 5$ in the $N$-domain and $\beta 11, \beta 14$, and $\beta 13$ in the $C$-domain) (Figure 7B). Around the $\beta$-sandwich core, consecutive $\alpha$-helices $(\alpha 1, \alpha 2$, $\alpha 3, \alpha 30$, and $\alpha 4$ in the $N$-domain and $\alpha 6, \alpha 7, \alpha 8$, and $\alpha 9$ in the $C$-domain) and one additional $\alpha$-helix ( $\alpha 5$ in the $N$-domain and $\alpha 10$ in the $C$-domain) are arranged. The $C$-domain has a large cleft, which NAD binds, similar to that of the $N$-domain (Figure 7B). The structure of VIP2 and C2I was solved $[46,47]$. VIP2 and C2I were divided into two domains like Ia. The count of identical residues in these enzyme components was $20 \%$ in the $C$-terminal domain and only $10 \%$ in the well conserved $N$-terminal domain. However, when Ia was superposed on VIP2, their $C$-domains were found to be quite similar in structure, but not their $N$-domains. It has been reported that the $N$-domains of C2I and Ia interact with the binding component, $\mathrm{C} 2 \mathrm{II}$ and $\mathrm{Ib}$, respectively [5]. Therefore, it is possible that the difference in structure of the $N$-domain reflects the difference in the binding component. It appears that the $\mathrm{N}$ - and $\mathrm{C}$-domains of these enzyme components play an important role in the binding to substrate and the binding component, and catalytic activity, respectively. 
Figure 7. Structure of Ia. (A) Structure of Ia with NADH (B) The $N$-domain and $C$-domain of Ia.

(A)

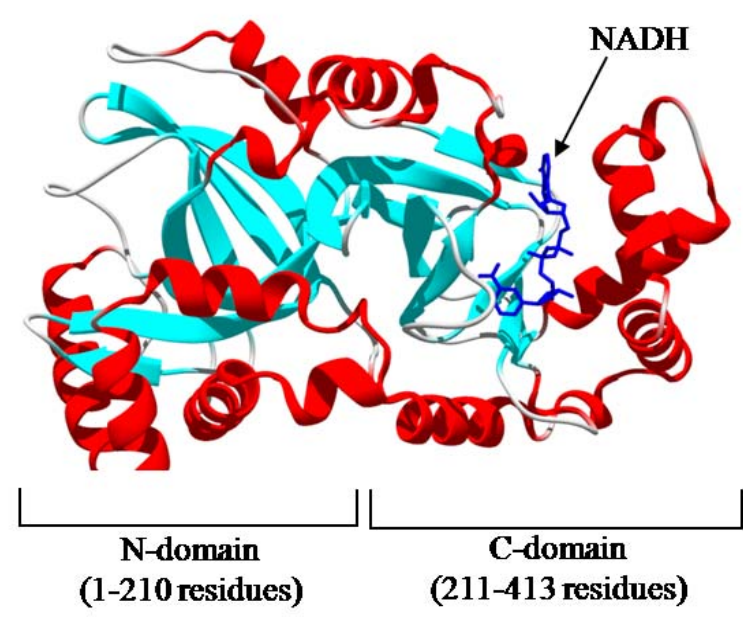

Red ribbon: $\alpha$-helices, sky blue ribbon: $\beta$-strands
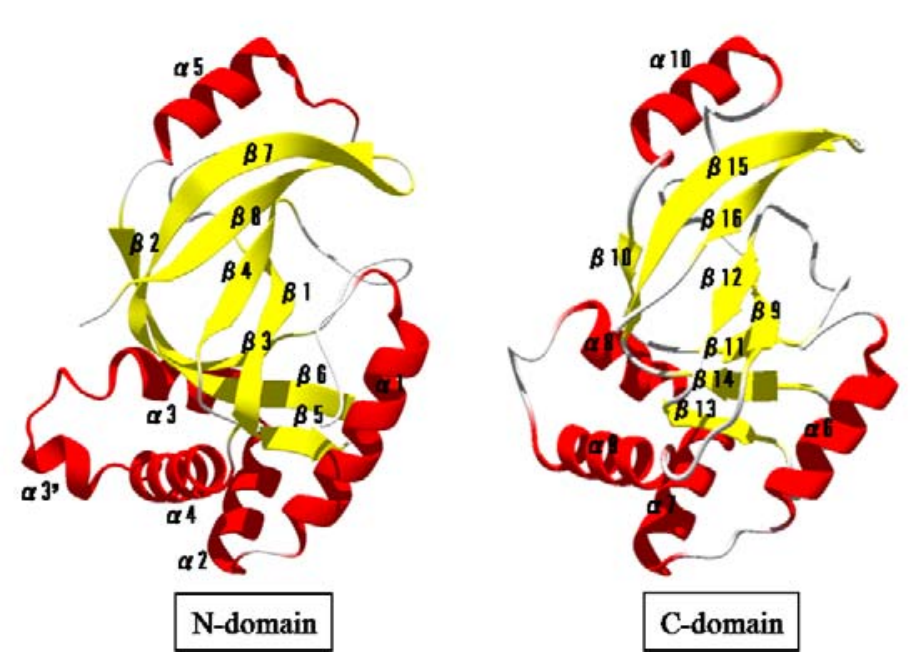

The crystal structure of Ia with NADH showed that the nicotinamide ring is clearly identifiable and nicotinamide is not cleaved $[45,48]$. NADH was highly folded in the cavity and formed a ring. As shown in Figure 8, N7N of the carboxyamide group on nicotinamide and O1N of the nicotinamide mononucleotide moiety (NMN) phosphate formed a hydrogen bond. Arg-295 formed a hydrogen bond

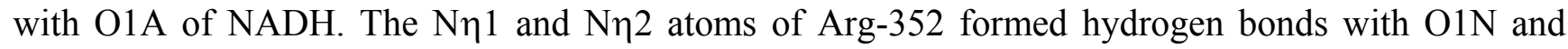
$\mathrm{O} 2 \mathrm{~N}$ of NADH, respectively. Oع2 of Glu-380 formed a hydrogen bond with $\mathrm{O} 2$ ' $\mathrm{N}$ of the nicotinamide ribose in the NMN. All of these hydrogen bonds appear important in making the NMN conformation compact and ring-like [45,48].

Residues located near the NADH-binding site in the $C$-domain of Ia have structural similarity to those in the $C$-domain of VIP. Tyr-246, Tyr-251, Asn-255, Arg-295, Glu-301, Ser-338, Phe-349, Arg-352, Glu-378, and Glu-380 in Ia are conserved, compared with those in VIP. A kinetic analysis of amino acid residues in the cavity showed that Tyr-251, Phe-349 and Arg-352 in Ia are required for binding to $\mathrm{NAD}^{+}[45,48]$. Thus, they are considered essential for ADP-ribosylating activity. One difference in the catalytic $C$-domain between Ia and VIP2 is in the occluding loop (372-380) that overlaps the so-called ARTT motif (373-PGYAGEYE-380), which includes the EXE motif (Glu-378 and Glu-380). Glu-378 and Glu-380 on the loop play an essential role in the catalytic activity of ADP-ribosylation [27], as mentioned above. While Glu-380 in Ia had the same conformation as that in VIP2, the orientation of Glu-378 located on the flexible loop differed between Ia and VIP2. Furthermore, $\mathrm{NAD}^{+}$-induced conformational change of the ARTT motif was seen in $\mathrm{C} 3$ toxin, which ADP-ribosylates Rho [49,50]. Therefore, it is likely that the ARTT motif is important for recognition of substrates. 
Figure 8. Distance between amino acid residues of the $C$-domain of Ia and NADH.

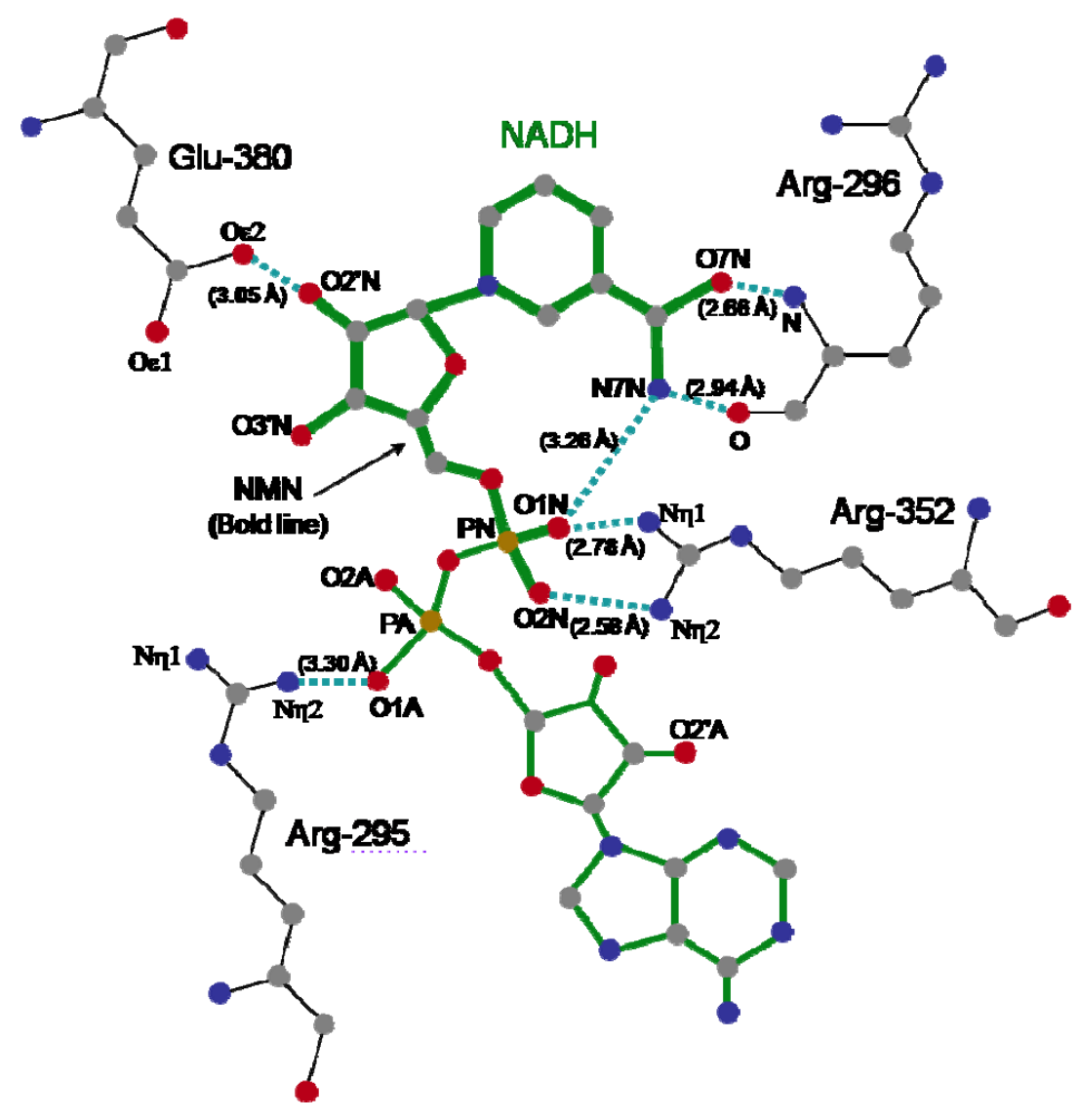

When the $C$-domain of Ia was superimposed on that of DT, the $\beta$-sheet fitted that of DT well, but the $\alpha$-helical part fitted less well, and amino acid residues in the catalytic site of Ia were superposed on those of DT [48]. Therefore, it appeared that Arg-295, Glu-380 and Phe-349 in Ia are arranged almost identically to His-21, Glu-148 and Tyr-65 in DT, and the STS motif near the folded NAD ${ }^{+}$in Ia is replaced by a YST sequence in DT $[45,48]$. From these findings it is concluded that toxins with ADP-ribosylating activity have a common basic tertiary structure consisting of a $\beta$-sandwich.

We reported the crystal structure of Ia-actin in a complex with the nonhydrolyzable NAD analog $\beta T A D$, at a resolution of $2.8 \AA$ [51] (Figure 9). Arg-177 and Asp-179 in G-actin are in close proximity to $\beta$ TAD and Glu-378 of Ia. The shape of bound $\beta$ TAD is similar to that of NADH, which assumes an NMN ring-like conformation. The structure indicated that Ia recognized actin via five loops around $\mathrm{NAD}^{+}$: loop I (Tyr-60-Tyr-62), loop II (active-site loop), loop III, loop IV (PN loop), and loop V (ADP-ribosylating turn-turn loop) (Figure 10 and 11). Furthermore, Glu-378 in the EXE is in close proximity to Arg-177 in actin.

The actin-binding interface of Ia was compared with that of other actin-binding proteins, including gelsolin [52], vitamin D-binding protein [53], DNaseI [54], and profilin [55]. DNaseI binds with high affinity to subdomains II and IV of actin, whereas the other three proteins bind to subdomains I and III. Ia mainly binds to actin through subdomains I, III, and IV, which cover its surface in the region that includes Arg-177 (Figure 12). The structure of the actin-Ia- $\beta$ TAD complex showed that the recognition interface of Ia differes from that of other known actin-binding proteins [51]. 
Figure 9. Ia-actin-TAD complex.

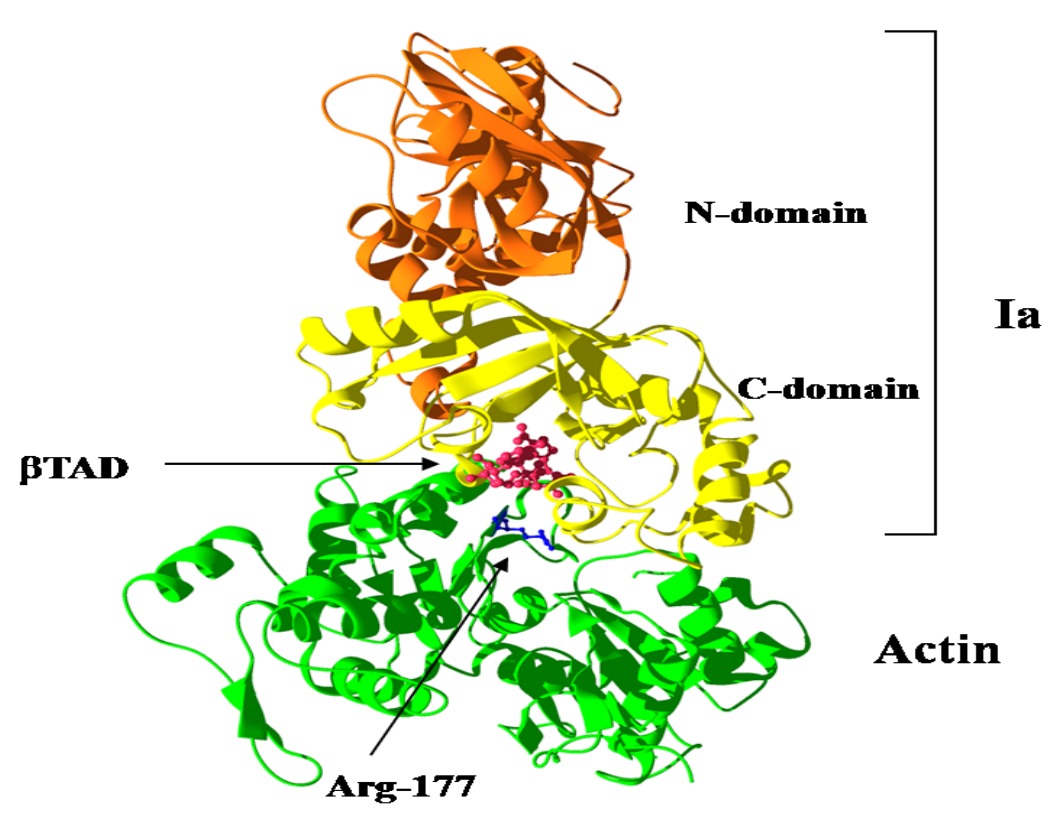

Figure 10. Sequence alignment of ADP-ribosylating toxins. Red circle: Amino acid residues in the expected actin binding region. Blue line: interface between the $\mathrm{N}$ and $\mathrm{C}$ domains. Secondary structure elements are coded as follows, $\beta$ sheet: yellow bars, $\alpha$ helices: magenta bars.

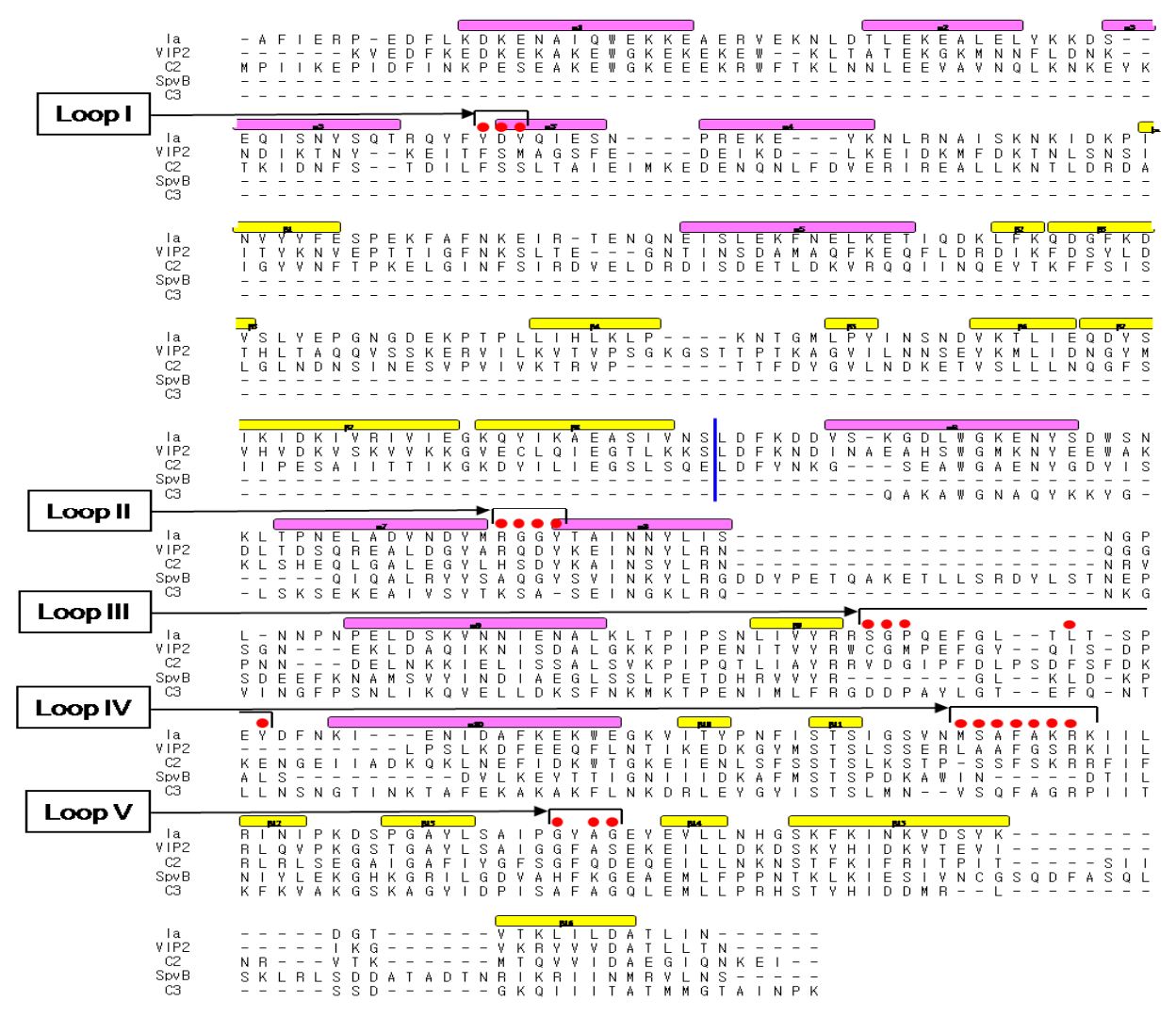


Figure 11. The loops in Ia involved in recognizing actin. Roman numerals (I-V) show the five actin-binding loops in Ia.

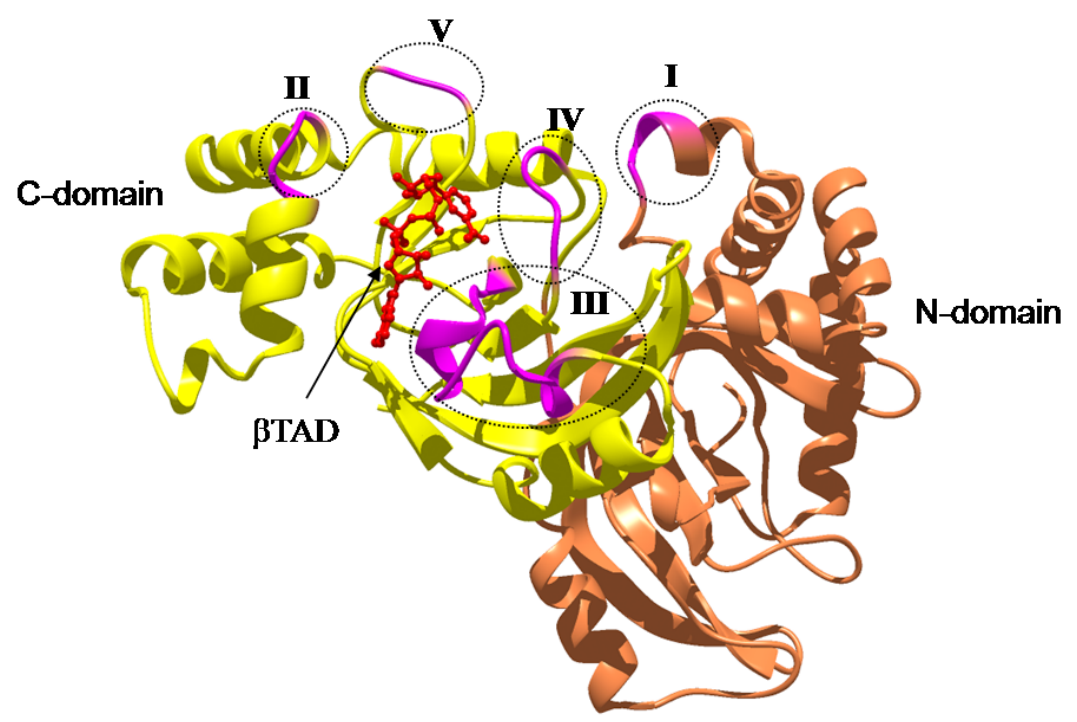

Figure 12. Comparison of the molecular recognition interface of Ia and actin-binding proteins. Actin (green) and Ia (magenta) were shown.
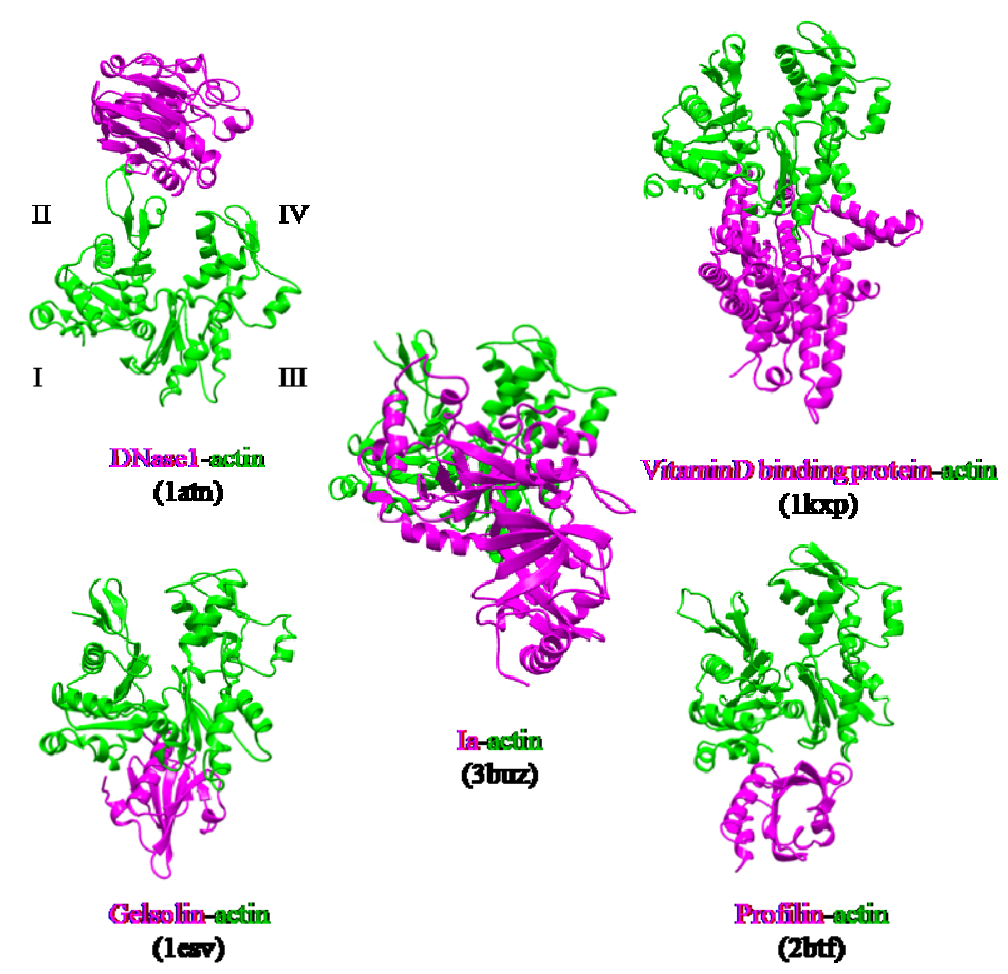

We investigated the role of amino acid residues, Asp-61, Tyr-62, Arg-248, Tyr-306 and Tyr-311, at the interface between Ia and actin [51]. The replacement of Tyr-62 in loop I and Arg-248 in loop II with alanine resulted in a drastic reduction of ARTase activity, but not NADase activity. However, the replacement of Asp-61 in loop I and Tyr-306 and Tyr-311 in loop III with alanine had little effect on ADP-ribosylating activity and cytotoxicity. Deletion of the $\mathrm{N}$ domain caused a drastic decrease in 
ARTase activity, whereas $>80 \%$ of the NADase activity was retained, suggesting the $N$ domain of Ia to be important for binding to actin. From these results, it was concluded that loops I and II of Ia play an important in binding to actin. On the other hand, the structure of actin within the complex was relatively unchanged from its monomeric form. Furthermore, binding of Ia to actin induced a subtle shift of loops II, IV, and V of Ia, suggesting that these loops play an important role in the recognition of actin. Therefore, it appears that actin is recognized by loops III, IV and V of Ia through not only ionic interaction, but also van der Waals interaction, based on the complementary shape. This feature might be common to all type IV ADP-ribosylating toxins that consist of two domains [51].

Two different mechanisms could be considered for the ADP-ribosylation of Arg-177 in actin by ADP-ribosylating toxins, the SN1- and SN2-reactions [45,46]. The positively charged Arg-295 and Arg-352 interact electrostatically with a phosphodiester group of $\mathrm{NAD}^{+}$and contribute to the highly folded conformation of NMN. The specific conformation appears to induce a shift in equilibrium toward the production of oxocarbenium cations from $\mathrm{NAD}^{+}$. A crystallographic analysis of the Ia-actin- $\beta$ TAD complex showed the distance between $\mathrm{NC} 1$ of $\mathrm{N}$-ribose and the guanidyl nitrogen of Arg-177 to be $>8 \AA$ (Figure 13A), which might make a direct nucleophilic attack impossible via the $\mathrm{SN} 2$ reaction. Even if the reaction proceeds via the SN1 mechanism, it is unclear how this distance is shortened. Therefore, it appears reasonable that the SN1 reaction occurs via the production of two intermediates: an oxocarbenium ion intermediate and a cationic intermediate (Figure 13B) [51]. After the oxocarbenium ion intermediate is produced, the NP-NO5 rotation of ADP ribose permits the $N$-ribose access to Arg-177. This second cationic intermediate allows the NC1 of $N$-ribose to approach the guanidyl nitrogen of Arg-177 to within a distance of $3.4 \AA$. (Figure 13B and C). In this model, Asp-179 of actin plays a stabilizing role by making contact with the $N$-ribose 2' $\mathrm{OH}$ (Figures $12 \mathrm{~B}$ and $\mathrm{C}$ ). Finally, the nucleophile Arg-177 of actin, which associates with Glu-378 of Ia, attacks NC1 of the oxocarbenium ion, leading to ADP-ribosylation. We reported that Tyr-251 binds to actin [45]. The structure shows that this residue is in close proximity to Asp-179 of actin. It therefore appears that Tyr-251 guides the NP-NO5 rotation to position the $N$-ribose. Furthermore, Tyr-251 might play a role in catching the oxocarbenium ion before it is transferred to the guanidyl nitrogen of Arg- 177 . Interestingly, it is possible that the residues around the $N$-ribose create a negative environment to protect the oxocarbenium cation and to prevent an unfavorable reaction with water; these comprise Tyr-246, Tyr-251, Ser-338, Tyr-375, Glu-378, and Glu-380. In addition, there is an open space within the interface of the complex, suggesting that it does not prevent reactions with water. The space makes it possible to rotate $\mathrm{N}$-ribose, thereby allowing $\mathrm{NC1}$ of the oxocarbenium ion to come sufficiently close to react with the guanidyl nitrogen of Arg-177. 
Figure 13. Mechanism for ADP-ribosylation of actin by Ia.

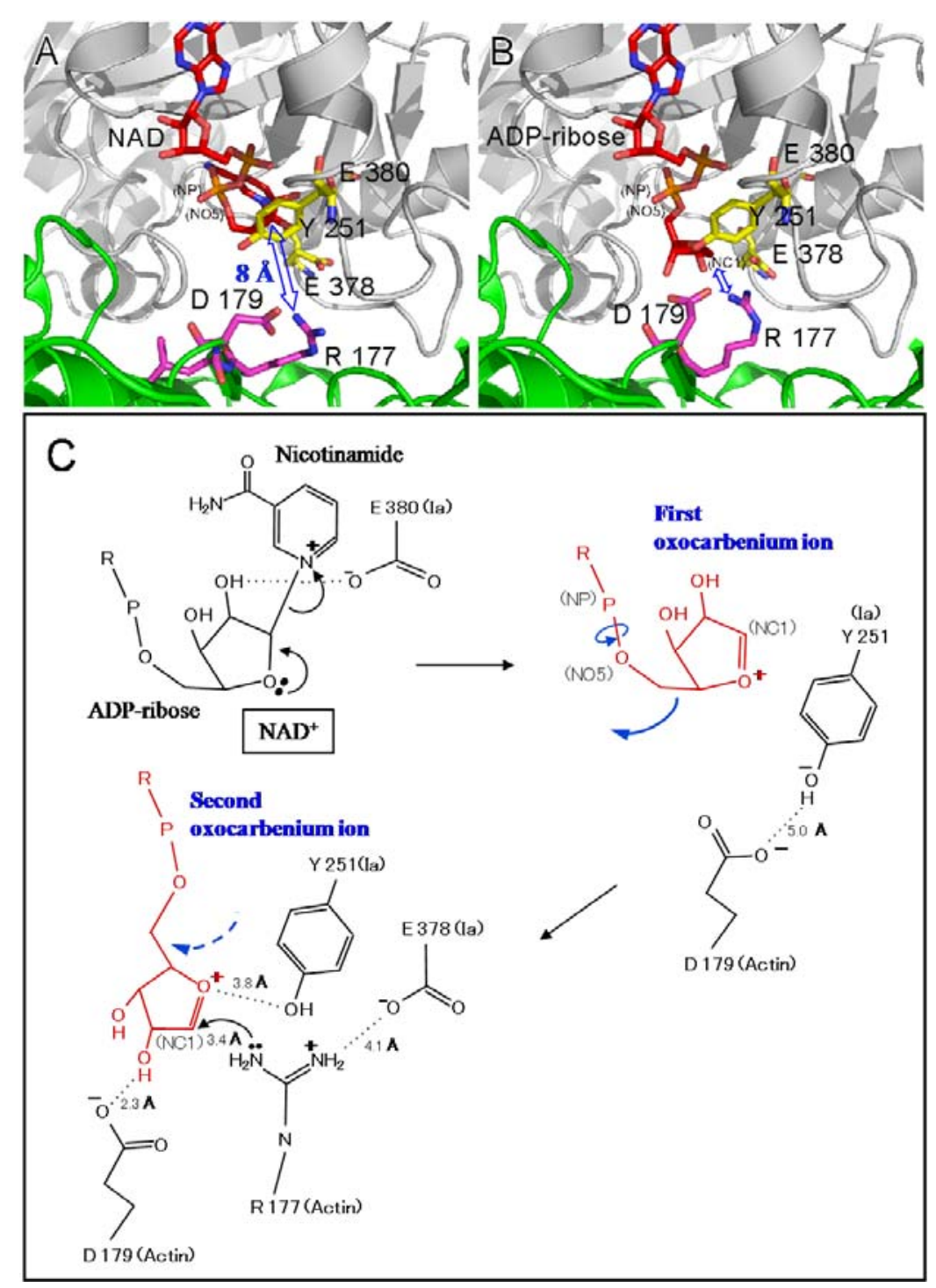

We propose a SN1 mechanism for the Arg-177 ADP-ribosylation that involves the following three steps: generation of the first oxocarbenium intermediate by the cleavage of nicotinamide, rotation to form the second cationic intermediate around the NP-NO5 bond through the release of the conformational strain, and nucleophilic attack of Arg-177 activated by the interaction with Glu-378 [51].

\section{Mode of Action}

Administration of Ib to mice given anti-Ia antiserum within 120 min after the intravenous injection of Ia did not cause death [11]. The result shows that the anti-Ia antiserum reacted with Ia in vivo until at least $120 \mathrm{~min}$ after the intravenous injection of Ia, suggesting that Ia remains free in the blood stream in the absence of $\mathrm{Ib}$. Administration of Ia to mice given anti-Ib antiserum $5 \mathrm{~min}$ after the injection of $\mathrm{Ib}$ resulted in death, indicating that the antiserum was unable to neutralize the activity under the conditions. It therefore appears that once it binds to the receptor, Ib proceeds immediately to a stage which can no longer be inhibited by the antiserum. These results suggest that Ia binds to the 
oligomer of $\mathrm{Ib}$ and penetrates the target tissue [11]. Dermonecrosis was observed at the site of injection of $\mathrm{Ib}$, but not Ia, when $\mathrm{Ia}$ and $\mathrm{Ib}$ were injected intradermally at separate sites in guinea pigs. Furthermore, the intraperitoneal injection of $\mathrm{Ia}$ and $\mathrm{Ib}$ after the intradermal injection of $\mathrm{Ib}$ and $\mathrm{Ia}$, respectively, resulted in dermonecrosis at the site of intradermal injection of $\mathrm{Ib}$, but not the intradermal injection of Ia. These results indicate that Ia is free in the blood-stream and Ib binds to a receptor in the skin, suggesting the migration of Ia to the site of injection of $\mathrm{Ib}$ to be essential to the dermonecrotic activity induced by separate injections of the two components [11]. Accordingly, it appears that Ia binds to Ib bound to target tissues, resulting in lethal effects and dermonecrosis.

The receptor-bound PA is reported to be internalized by receptor-mediated endocytosis [56]. Milne et al. [57] found that PA formed oligomers during the intoxication of mammalian cells. The Aktories group has reported that C2II toxin, which resembles PA, forms an oligomer during intoxication [58]. However, it has been reported that $\mathrm{Ib}$ possesses no activity [6,9,59]. Knapp et al. reported that $\mathrm{Ib}$ formed a single-channel in artificial lipid bilayers and Vero cells [60]. Recently, we reported that $\mathrm{Ib}$ specifically binds to Vero cells and induces the release of $\mathrm{K}^{+}$from the cells in a dose- and time-dependent manner, showing that $\mathrm{Ib}$ forms ion-permeable channels in Vero cells without causing death [41]. Therefore, it is apparent that $\mathrm{Ib}$ also possesses biological activity. SDS-PAGE revealed that the migration of the $\mathrm{Ib}$ oligomer formed by incubation of Vero cells with $\mathrm{Ib}$ at $37^{\circ} \mathrm{C}$ was identical to that of the Ib heptamer isolated from the purified Ib preparation, showing that $\mathrm{Ib}$ forms an oligomer on the cell membrane [41]. The time course of $\mathrm{K}^{+}$'s release from the cells treated with $\mathrm{Ib}$ coincided with that of the oligomer's formation. On the other hand, when Vero cells were incubated with oligomers isolated from the purified $\mathrm{Ib}$ preparation, no release of $\mathrm{K}^{+}$was detected, rounding of the cells was not observed in the presence of Ia, and the oligomers bound to the cells were hydrolyzed by treatment with pronase. The oligomer formed from the monomer in membranes of the cells was not hydrolyzed by the enzyme. These observations imply that the monomer of $\mathrm{Ib}$ binds to the cell and forms a functional oligomer in the membrane [41].

PA and diphtheria toxin were shown to be translocated into the cytosol from an endosomal compartment. C2 toxin was reported to enter cells through receptor-mediated endocytosis [59]. Furthermore, Barth et al. [58] reported that $\mathrm{C} 2$ toxin is released into the cytosol after acidification of the endosomal compartment. Considine and Simpson [59] suggested the internalization of Ib by receptor-mediated endocytosis to be essential to the toxicity of iota-toxin. Blocker et al. reported that treatment with Ib plus Ia in the presence of bafilomycin, methylamine and ethylamine, which prevents acidification of endosomal vesicles, caused no change in cells and, in addition, did not cause ADP-ribosylation of actin in the cytosol [61]. Ib was trans-cytosed and permanently exposed on surface of the opposite cell or continuously recycled between an endosomal compartment and the cell surface [23]. We obtained evidence of the cellular routing of Ib. After Vero cells were treated with these inhibitors, Ib induced the release of $\mathrm{K}^{+}$from the cells, and the oligomer formed on the cells and was inserted into the endosomal membranes. These observations suggest that $\mathrm{Ib}$ forms ion-permeable channels in the membranes (functional oligomer) and that $\mathrm{Ib}$ is endocytosed after binding to the cell in the absence of Ia. 
Recently, lipid rafts have been reported to act as surface platforms during signal transduction and endocytosis [62]. In addition, some toxins [63,64], bacteria [65], and viruses [66] have been shown to enter cells via lipid rafts. We investigated whether or not the oligomer of $\mathrm{Ib}$ accumulates in lipid rafts and Ia bound to the oligomer enters the cell. The monomer of $\mathrm{Ib}$ was detected in the Triton $\mathrm{X}-100$-soluble and -insoluble fractions, the whole membrane, of cells incubated with $\mathrm{Ib}$ at $4{ }^{\circ} \mathrm{C}$, suggesting that the receptor of $\mathrm{Ib}$ is distributed around cytoplasmic membranes. Thus, it is unlikely that the receptor is confined to lipid rafts. The oligomer of $\mathrm{Ib}$ was detected in lipid rafts after incubation of the washed cells at $37^{\circ} \mathrm{C}$, suggesting that $\mathrm{Ib}$ bound to the receptor is gathered in lipid rafts, and the oligomer is formed there. In addition, the $C$-terminal region of $\mathrm{Ib}$ (Ib421-664) blocked the binding of $\mathrm{Ib}$ to the cells, as reported by Marvaud et al. [67], and was detected in lipid rafts from MDCK cells. It therefore appears that the $C$-terminal region of $\mathrm{Ib}$ binds to a receptor that is distributed over the entire membrane of the cell, that a receptor which is linked with Ib gathers in lipid rafts at $37^{\circ} \mathrm{C}$, and that $\mathrm{Ib}$ forms oligomers in the lipid rafts [43]. The treatment of MDCK cells with M $\beta C D$ reduced the cholesterol content of lipid raft fractions, the binding of $\mathrm{Ib}$ to the cells, and the rounding activity induced by Ia plus Ib. Therefore, it has been speculated that the functional properties of lipid rafts that are relevant to the intracellular trafficking of $\mathrm{Ib}$ may be especially susceptible to treatment with $\mathrm{M} \beta \mathrm{CD}$. It was reported that the disruption or depletion of membrane-associated cholesterol causes major changes in the function and/or distribution of raft-associated membrane components [68]. It therefore appears that a function of lipid rafts is to gather Ib so that it forms oligomers and enters into cells [43].

The internalization of iota-toxin is mediated through lipid rafts (cholesterol-rich microdomains) at the plasma membrane, suggesting that the lipid rafts contain all of the components necessary for the mediation of the toxin's endocytosis. Gibert et al. reported that after internalization, it was found that iota-toxin was not routed to the Golgi apparatus and translocation of the Ia occurs early endosomes [69]. The findings that $\mathrm{Ib}$ is concentrated in lipid rafts, where it forms oligomers, and that it induces endocytosis have provided useful information on the cytotoxicity induced by bacterial toxins.

\section{Conclusions}

Iota-toxin enters host cells and induces toxicity by exploiting the cell's endogenous pathways as follows (Figure 14). (1) Ib binds to a receptor at plasma membranes, then moves to lipid-rafts and forms a heptamer, (2) the $\mathrm{N}$-terminal domain of Ia binds to the $\mathrm{Ca}^{2+}$-binding motif in the $\mathrm{N}$-terminal region of $\mathrm{Ib}$, (3) Ia bound to the Ib oligomer is internalized in cells by receptor-mediated endocytosis, (4) the complex of $\mathrm{Ia}$ and $\mathrm{Ib}$ is transported to the early endosomes, where acidification promotes cytosolic entry of Ia, and 5) the $C$-domain and part of the $N$-domain of Ia bind to G-actin in the cytosol and ADP-ribosylate it, thereby blocking the polymerization of actin, and eventually intoxicating cells. 
Figure 14. Model for internalization of iota-toxin into cells.

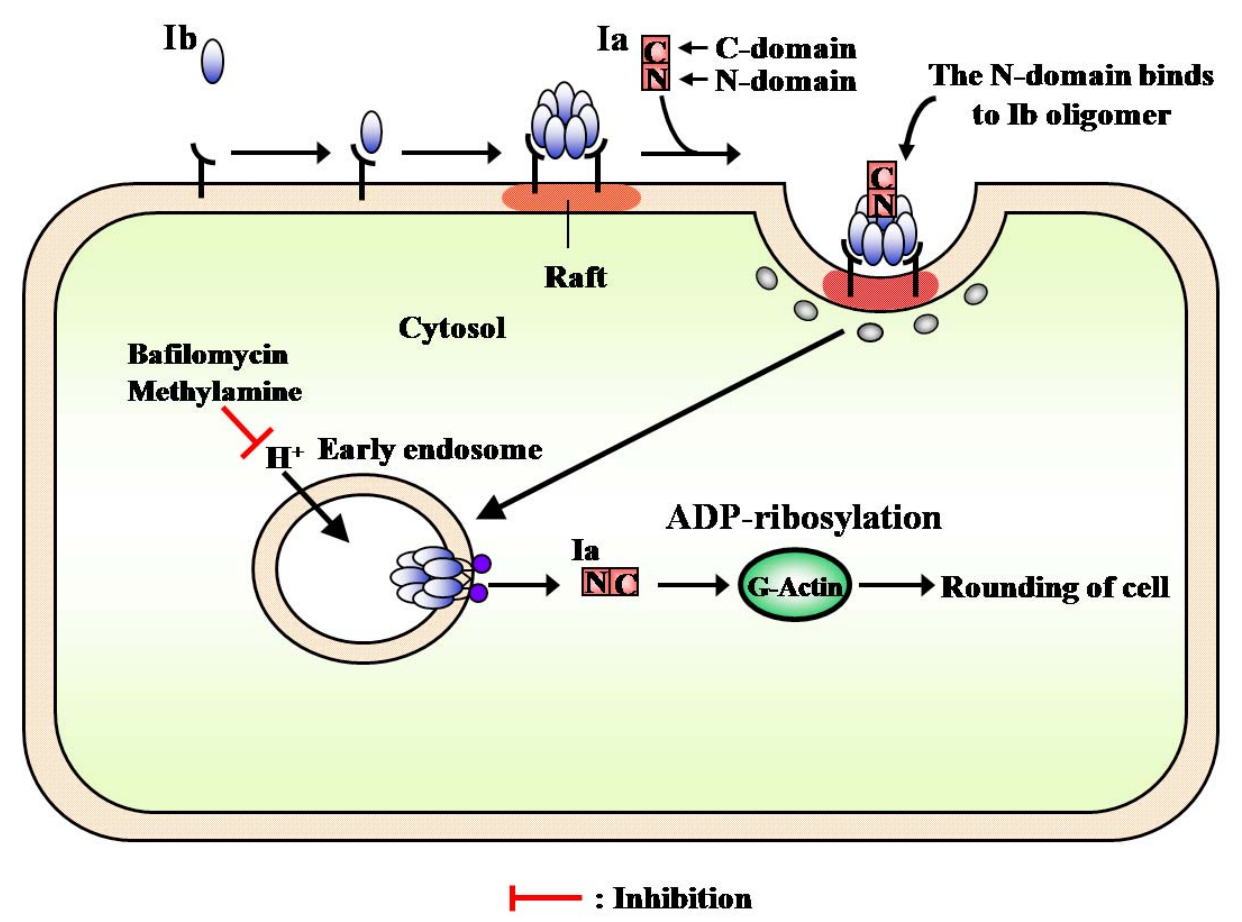

\section{References}

1. McDonel, J.L. Pharmacology of Bacterial Toxins; Pergamon Press: New York, NY, USA, 1986; pp. 477-517.

2. Sakurai, J. Toxins of Clostridium perfringens. Rev. Med. Microbiol. 1995, 6, 175-185.

3. Sakurai, J.; Nagahama. M.; Ochi, S. Major toxins of Clostridium perfringens. J. Toxicol-_Toxin Rev. 1997, 16, 195-214.

4. Songer, J.G. Clostridial enteric diseases of domestic animals. Clin. Micr obiol. Rev. 1996, 9, 216-234.

5. Barth, H.; Aktories, K.; Popoff, M.R.; Stiles, B.G. Binary bacterial toxins: Biochemistry, biology, and applications of common Clostridium and Bacillus proteins. Microbiol. Mol. Biol. Rev. 2004, $68,373-402$.

6. Aktories, K.; Wegner, A. ADP-ribosylation of actin by clostridial toxins. J. Cell Biol. 1989, 109, 1385-1387.

7. Gibert, M.; Petit, L.; Raffestin, S.; Okabe, A.; Popoff, M.R. Clostridium perfringens iota-toxin requires activation of both binding and enzymatic components for cytopathic activity. Infect. Immun. 2000, 68, 3848-3853.

8. Bosworth, T.J. On a new type of toxin produced by Clostridium welchii. J. Comp. Pathol. 1943, 53, 245-255.

9. Stiles, B.G.; Wilkins, T.D. Purification and characterization of Clostridium perfringens iota toxin: Dependence on two nonlinked proteins for biological activity. Infect. Immun. 1986, 54, 683-688.

10. Perelle, S.; Gibert, M.; Boquet, P.; Popoff, M.R. Characterization of Clostridium perfringens iota-toxin genes and expression in Escherichia coli. Infect. Immun. 1993, 61, 5147-5156. 
11. Sakurai, J.; Kobayashi, K. Lethal and dermonecrotic activities of Clostridium perfringens iota toxin: Biological activities induced by cooperation of two nonlinked components. Microbiol. Immunol. 1995, 39, 249-253.

12. Krueger, K.M.; Barbieri, J.T. The family of bacterial ADP-ribosylating exotoxins. Clin. Microbiol. Rev. 1995, 8, 34-47.

13. Holbourn, K.P.; Shone, C.C.; Acharya, K.R. A family of killer toxins. Exploring the mechanism of ADP-ribosylating toxins. FEBS J. 2006, 273, 4579-4593.

14. Gill, D.M.; Meren, R. ADP-ribosylation of membrane proteins catalyzed by cholera toxin: Basis of the activation of adenylate cyclase. Proc. Natl. Acad. Sci. USA. 1978, 75, 3050-3054.

15. Katada, T.; Ui, M. Direct modification of the membrane adenylate cyclase system by islet-activating protein due to ADP-ribosylation of a membrane protein. Proc. Natl. Acad. Sci. USA. 1982, 79, 3129-3133.

16. Moss, J.; Garrison, S.; Oppenheimer. N.J.; Richardson, S.H. NAD-dependent ADP-ribosylation of arginine and proteins by Escherichia coli heat-labile enterotoxin. J. Biol. Chem. 1979, 254, $6270-6272$.

17. van Ness, B.G.; Howard, J.B.; Bodley, J.W. ADP-ribosylation of elongation factor 2 by diphtheria toxin. Isolation and properties of the novel ribosyl-amino acid and its hydrolysis products. J. Biol. Chem. 1980, 255, 10717-10720.

18. Deng, Q.; Barbieri, J.T. Molecular mechanisms of the cytotoxicity of ADP-ribosylating toxins. Ann. Rev. Microbiol. 2008, 62, 271-288.

19. Aktories, K.; Weller, U.; Chhatwal, G.S. Clostridium b otulinum type C produces a novel ADP-ribosyltransferase distinct from botulinum C2 toxin. FEBS Lett. 1987, 212, 109-113.

20. Aktories, K.; Bärmann, M.; Ohishi, I.; Tsuyama S.; Jakobs, K.H.; Habermann, E. Botulinum C2 toxin ADP-ribosylates actin. Nature 1986, 322, 390-392.

21. Popoff, M.R.; Rubin, E.J.; Gill, D.M.; Boquet, P. Actin-specific ADP-ribosyltransferase produced by a Clostridium difficile strain. Infect. Immun. 1988, 56, 2299-2306.

22. Popoff, M.R.; Boquet, P. Clostridium spiroforme toxin is a binary toxin which ADP-ribosylates cellular actin. Biochem. Biophys. Res. Commun. 1988, 152, 1361-1368.

23. Richard, J.F.; Mainguy, G.; Gibert, M.; Marvaud, J.C.; Stiles, B.G.; Popoff, M.R. Transcytosis of iota-toxin across polarized CaCo-2 cells. Mol. Microbiol. 2002, 43, 907-917.

24. Hilger, H.; Pust, S.; von Figura, G.; Kaiser, E.; Stiles, B.G.; Popoff, M.R.; Barth, H. The long-lived nature of Clostridium perfringens iota toxin in mammalian cells induces delayed apoptosis. Infect. Immun. 2009, 77, 5593-5601.

25. Domenighini, M.; Rappuoli. R. Three conserved consensus sequences identify the NAD-binding site of ADP-ribosylating enzymes, expressed by eukaryotes, bacteria and T-even bacteriophages. Mol. Microbiol. 1996, 21, 667-674.

26. Carpusca, I.; Jank, T.; Aktories, K. Bacillus sphaericus mosquitocidal toxin (MTX) and pierisin: The enigmatic offspring from the family of ADP-ribosyltransferases. Mol. Microbiol. 2006, 62, 621-630. 
27. Nagahama, M.; Sakaguchi, Y.; Kobayashi, K.; Ochi, S.; Sakurai, J. Characterization of the enzymatic component of Clostridium perfringens iota-toxin. J. Bacteriol. 2000, 182, 2096-2103.

28. Perelle, S.; Domenighini, M.; Popoff, M.R. Evidence that Arg-295, Glu-378, and Glu-380 are active-site residues of the ADP-ribosyltransferase activity of iota toxin. FEBS Le tt. 1996, 395, 191-194.

29. Barth, H.; Preiss, J.C.; Hofmann, F.; Aktories, K. Characterization of the catalytic site of the ADP-ribosyltransferase Clostridium botulinum C2 toxin by site-directed mutagenesis. J. Biol. Chem. 1998, 273, 29506-29511.

30. Domenighini, M.; Montecucco, C.; Ripka, W.C.; Rappuoli, R. Computer modeling of the NAD binding site of ADP-ribosylating toxins: Active-site structure and mechanism of NAD binding. Mol. Microbiol. 1991, 5, 23-31.

31. Radke, J.; Pederson, K.J.; Barbieri, J.T. Pseudomonas aeruginosa exoenzyme S is a biglutamic acid ADP-ribosyltransferase. Infect. Immun. 1999, 67, 1508-1510.

32. Damme, J.; Jung, M.; Hofmann, F.; Just, I.; Vandekerckhove, J.; Aktories, K. Analysis of the catalytic site of the actin ADP-ribosylating Clostridium perfringens iota toxin. FEBS Lett. 1996, 380, 291-295.

33. Cieplak, W.; Mead, D.J.; Messer, R.J.; Grant, C.C. Site-directed mutagenic alteration of potential active-site residues of the A subunit of Escherichia coli heat-labile enterotoxin. Evidence for a catalytic role for glutamic acid 112. J. Biol. Chem. 1995, 270, 30545-30550.

34. Hara, N.; Tsuchiya, M.; Shimoyama, M. Glutamic acid 207 in rodent T-cell RT6 antigens is essential for arginine-specific ADP-ribosylation. J. Biol. Chem. 1996, 271, 29552-29555.

35. Aktories, K.; Jung, M.; Böhmer, J.; Fritz, G.; Vandekerckhove, J.; Just, I. Studies on the active-site structure of C3-like exoenzymes: Involvement of glutamic acid in catalysis of ADP-ribosylation. Biochimie. 1995, 77, 326-332.

36. Petosa, C.; Collier, R.J.; Klimpel, K.R.; Leppla, S.H.; Liddington, R.C. Crystal structure of the anthrax toxin protective antigen. Nature 1997, 385, 833-838.

37. Schleberger, C.; Hochmann, H.; Barth, H.; Aktories, K.; Schulz, G.E. Structure and action of the binary C2 toxin from Clostridium botulinum. J. Mol. Biol. 2006, 364, 705-715.

38. Gupta, P.K.; Chandra, H.; Gaur, R.; Kurupati, R.K.; Chowdhury, S.; Tandon, V.; Sigh, Y.; Maithal, $\mathrm{K}$. Conformational fluctuations in anthrax protective antigen: A possible role of calcium in the folding pathway of the protein, FEBS Lett. 2003, 554, 505-510.

39. Gao-Sheridan, S.; Zhang, S.; Collier, R.J. Exchange characteristics of calcium ions bound to anthrax protective antigen. Biochem. Biophys. Res. Commun. 2002, 300, 61-64.

40. Leppla, S.A. The Compr ehensive Sour cebook of Bacterial Pr otein Toxins, $2^{\text {nd }}$ ed.; Academic Press: San Diego, CA, USA, 1991; pp. 243-261.

41. Nagahama, M.; Nagayasu, K.; Kobayashi, K.; Sakurai, J. Binding component of Clostridium perfringens iota-toxin induces endocytosis in vero cells. Infect. Immun. 2002, 70, 1909-1914.

42. Kobayashi, K.; Nagahama, M.; Ohkubo, N.; Kojima, T.; Shirai, H.; Iwamoto, S.; Oda, M.; Sakurai, J. Role of $\mathrm{Ca}^{2+}$-binding motif in cytotoxicity induced by Clostridium perfringens iota-toxin. Microb. Pathog. 2008, 44, 265-270. 
43. Nagahama, M.; Yamaguchi, A.; Hagiyama, T.; Ohkubo, N.; Kobayashi, K.; Sakurai, J. Binding and internalization of Clostridium perfringens iota-toxin in lipid rafts. Infect. Immun. 2004, 72, 3267-3275.

44. Tsuge, H.; Nagahama, M.; Nishimura, T.; Sakaguchi, Y.; Katunuma, N.; Sakurai, J. Crystallization and preliminary X-ray studies of the Ia component of Clostridium perfringens iota toxin complexed with NADPH. J. Struct. Biol. 1999, 126, 175-177.

45. Tsuge, H.; Nagahama, M.; Nishimura, H.; Hisatsune J.; Sakaguchi, Y.; Itogawa, Y.; Katunuma, N.; Sakurai, J. Crystal structure and site-directed mutagenesis of enzymatic components from Clostridium perfringens iota-toxin. J. Mol. Biol. 2003, 325, 471-483.

46. Han, S.; Craig, J.A.; Putnam, C.D.; Carozzi, N.B.; Tainer, J.A. Evolution and mechanism from structures of an ADP-ribosylating toxin and NAD complex. Nat. Struct. Biol. 1999, 6, 932-936.

47. Schleberger, C.; Hochmann, H.; Barth, H.; Aktories, K.; Schulz, G.E. Structure and action of the binary C2 toxin from Clostridium botulinum. J. Mol. Biol. 2006, 384, 705-715.

48. Sakurai, J.; Nagahama, M.; Hisatsune, J.; Katunuma, N.; Tsuge, H. Clostridium perfringens iota-toxin, ADP-ribosyltransferase: Structure and mechanism of action. Adv. Enzyme Regul. 2003, 43, 361-377.

49. Menetrey, J.; Flatau, G.; Stura, E.A.; Charbonnier, J.B.; Gas, F.; Teulon, J.M.; Le Du, M.H.; Boquet, P.; Menez, A. NAD binding induces conformational changes in Rho ADP-ribosylating Clostridium botulinum C3 exoenzyme, J. Biol. Chem. 2002, 277, 30950-30957.

50. Han, S.; Arvai, A.S.; Clancy, S.B.; Tainer, J.A. Crystal structure and novel recognition motif of rho ADP-ribosylating C3 exoenzyme from Clostridium botulinum : Structural insights for recognition specificity and catalysis. J. Mol. Biol. 2001, 305, 95-107.

51. Tsuge, H.; Nagahama, M.; Oda, M.; Iwamoto, S.; Utsunomiya, H.; Marquez, V.E.; Katunuma, N.; Nishizawa, M.; Sakurai, J. Structural basis of actin recognition and arginine ADP-ribosylation by Clostridium perfringens iota-toxin. Proc. Natl. Acad. Sci. USA. 2008, 105, 7399-7404.

52. McLaughlin, P.J.; Gooch, J.T.; Mannherz, H.G.; Weeds, A.G. Structure of gelsolin segment 1-actin complex and the mechanism of filament severing. Nature. 1993, 364, 685-692.

53. Otterbein, L.R.; Cosio, C.; Graceffa, P.; Dominguez, R. Crystal structures of the vitamin D-binding protein and its complex with actin: Structural basis of the actin-scavenger system. Proc. Natl. Acad. Sci. USA. 2002, 99, 8003-8008.

54. Kabsch, W.; Mannherz, H.G.; Suck, D.; Pai, E.F.; Holmes, K.C. Atomic structure of the actin:DNase I complex. Nature 1990, 347, 37-44.

55. Schutt, C.E.; Myslik, J.C.; Rozycki, M.D.; Goonesekere, N.C.; Lindberg, U. The structure of crystalline profilin-beta-actin. Nature 1993, 365, 810-816.

56. Singh, Y.; Klimpel, K.R.; Arora, N.; Sharma, M.; Leppla, S.H. The chymotrypsin-sensitive site, FFD315, in anthrax toxin protective antigen is required for translocation of lethal factor. $J$. Biol. Chem. 1994, 269, 29039-29046.

57. Milne, J.C.; Furlong, D.; Hanna, P.C.; Wall, J.S.; Collier, R.J. Anthrax protective antigen forms oligomers during intoxication of mammalian cells. J. Biol. Chem. 1994, 269, 20607-20612. 
58. Barth, H.; Blocker, D.; Behlke, J.; BergsmafSchutter, W.; Brisson, A.; Benz, R.; Aktories, K. Cellular uptake of Clostridium botulinum $\mathrm{C} 2$ toxin requires oligomerization and acidification. $J$. Biol. Chem. 2000, 275, 16478-16483.

59. Considine, R.V.; Simpson, L.L. Cellular and molecular actions of binary toxins possessing ADP-ribosyltransferase activity. Toxicon. 1991, 29, 913-936.

60. Knapp, O.; Benz, R.; Gibert, M.; Marvaud, J.C.; Popoff, M.R. Interaction of Clostridium perfringens iota-toxin with lipid bilayer membranes. Demonstration of channel formation by the activated binding component $\mathrm{Ib}$ and channel block by the enzyme component Ia. J. Biol. Chem. 2002, 277, 6143-6152.

61. Blocker, D.; Behlke, J.; Aktories, K.; Barth, H. Cellular uptake of Clostridium perfringens binary iota-toxin. Infect. Immun. 2001, 69, 2980-2987.

62. Nabi, I.R.; Le, P.U. Caveolae/raft-dependent endocytosis. J. Cell Biol. 2003, 161, 673-677.

63. Nagahama, M.; Hayashi, S.; Morimitsu, S.; Sakurai, J. Biological activities and pore formation of Clostridium perfringens beta toxin in HL 60 cells. J. Biol. Chem. 2003, 278, 36934-36941.

64. Nagahama, M.; Hagiyama, T.; Kojima, T.; Aoyanagi, K.; Takahashi, C.; Oda, M.; Sakaguchi, Y.; Oguma, K.; Sakurai, J. Binding and internalization of Clostridium botulinum C2 toxin. Infect. Immun. 2009, 77, 5139-5148.

65. Duncan, M.J.; Shin, J.S.; Abraham, S.N. Microbial entry through caveolae: Variations on a theme. Cell Microbiol. 2002, 4, 783-791.

66. Chazal, N.; Gerlier, D. Virus entry, assembly, budding, and membrane rafts. Microbiol. Mol. Biol. Rev. 2003, 67, 226-237.

67. Marvaud, J.C.; Smith, T.; Hale, M.L.; Popoff, M.R.; Smith, L.A.; Stiles, B.G. Clostridium perfringens iota-toxin: Mapping of receptor binding and Ia docking domains on Ib. Infect. Immun. 2001, 69, 2435-2441.

68. Schraw, W.; Li, Y.; McClain, M.S.; van der Goot, F.G.; Cover, T.L. Association of Helicobacter pylori vacuolating toxin (VacA) with lipid rafts. J. Biol. Chem. 2002, 277, 34642-34650.

69. Gibert, M.; Marvaud, J.C.; Pereira, Y.; Hale, M.L.; Stiles, B.G.; Boquet, P.; Lamaze, C.; Popoff, M.R. Differential requirement for the translocation of clostridial binary toxins: Iota toxin requires a membrane potential gradient. FEBS Lett. 2007, 581, 1287-1296.

(C) 2009 by the authors; licensee Molecular Diversity Preservation International, Basel, Switzerland. This article is an open-access article distributed under the terms and conditions of the Creative Commons Attribution license (http://creativecommons.org/licenses/by/3.0/). 